\title{
The OECD Blue Book on Recombinant DNA Safety Considerations: it's influence on ISBR and EFSA activities
}

\author{
Joachim SCHIEMANN ${ }^{\star}$ \\ Federal Biological Research Centre for Agriculture and Forestry, Institute for Plant Virology, Microbiology and Biosafety, Braunschweig, \\ Germany
}

Biosafety regulatory frameworks are intended to serve as mechanisms for ensuring the safe use of biotechnology products without imposing unacceptable risk to human health or the environment, or unintended constraints to technology transfer. The OECD Blue Book on "Recombinant DNA Safety Considerations", setting out principles and concepts for handling genetically modified organisms safely outside of contained laboratory conditions, was a milestone in the history of biotechnology. The "Recombinant DNA Safety Considerations" definitively became the major resource for the formulation of national regulatory frameworks and international regulations, including the Cartagena Protocol.

As President of the International Society for Biosafety Research (ISBR) and member of the GMO Panel of the European Food Safety Authority (EFSA) I want to present my personal view on the influence the OECD Blue Book has had on the work of our society and the development of the Guidance document of the Scientific Panel on Genetically Modified Organisms for the Risk Assessment of Genetically Modified Plants and Derived Food and Feed (EFSA, 2004). These reflections are strongly linked with the memory of Rudolf Casper, who introduced me to the Blue Book's philosophy when I entered his institute in 1991. Prof. Dr. Rudolf Casper, who died shortly after his 75th birthday, was a pioneer in GMO biosafety research, the organizer of the 2nd International Symposium on Biosafety of Genetically Modified Organisms (ISBGMO) in Goslar, a great scientist, a great organizer and a wonderful human being.

A main prerequisite to improving the science-based discussion on GMO biosafety and the science-based decision-making for placing GMOs on the market is to focus and strengthen the voice of GMO biosafety research. The ISBR plays a key role in this process. The ISBR aims to promote scientifically sound biosafety research by improving communication among scientists who study plants, animals, and microbes with new characteristics due to altered DNA and produced using modern biotechnology. By fostering communication and technical exchange among experts, ISBR supports the science used in biosafety assessment processes, and promotes constructive dialogue on important science-based

*Corresponding author: J.Schiemann@BBA.DE biosafety issues associated with GMOs. Our forebears in the EU, US and elsewhere convened a Symposium in 1990 in Kiawah Island, North Carolina, entitled "Biological Monitoring of Genetically Engineered Plants and Microbes" reflecting the state of technology at the time. The second Symposium, entitled "The biosafety results of field tests of genetically modified plants and micro-organisms" was held in Goslar, Germany in 1992. Subsequent biennial Symposia were held in Monterey, USA (1994), Tsukuba, Japan (1996), Braunschweig, Germany (1998), Saskatoon, Canada (2000), Beijing, China (2002), Montpellier, France (2004) and Jeju, Korea (2006). These symposia have afforded the opportunity for stakeholders with diverse perspectives, interests, and areas of expertise to share information and exchange ideas on matters concerning the biosafety of GMOs. The symposium series is designed for academicians, policy makers, regulators, NGOs, and industry representatives interested in recent scientific research with GMOs. The theme of the 2006 symposium was: "Biosafety Research and Environmental Risk Assessment". As in previous symposia, the meeting focused on scientific findings that are relevant to regulatory decision-making worldwide. Since the unique value of our biennial symposia lies in providing an international context for dialogue between researchers and those involved in risk assessment and risk management, to link the 9th ISBGMO with a workshop celebrating the twentieth anniversary of the OECD guideline turned out to be an excellent idea. The full day OECD workshop provided a possibility to discuss all aspects of the Blue Book - how scientists, policy makers, and regulators see these guidelines today. 
Twenty years of the OECD Blue Book, 16 years of ISBGMOs. At the 2nd ISBGMO in 1992 in Goslar (Casper and Landsmann, 1992) a talk was presented by Bruna Teso (OECD) on "International Harmonization of Safety Principles for Biotechnology". Some ideas from the talk, still remarkably interesting for our current debate, will be cited in the following. "Historically, biotechnology is not the only example of a new technology that has raised concerns in the scientific community, the government and the public at large. However, it is the only one which was subjected not only to close scrutiny but also to strict oversight in the very early stages of its implementation. There is a general consensus within the scientific community that the risks posed by modern biotechnology are essentially the same in nature as those posed by conventional biotechnology and that they can also be assessed in a similar way. The safe record of the work involving recombinant organisms carried out in more than fifteen years in laboratories, industry and in some five hundred field experiments carried out to date, confirm and justify this positive view. The OECD Committee for Scientific and Technological Policy therefore created in 1983 a group of experts on safety. Its mandate was to identify scientific principles and criteria for the safe use of genetically modified organisms in industry, agriculture and in the environment. In light of the confused debate on the potential consequences of biotechnology going on at that time, the work of the OECD experts focused on the development of a rational and flexible approach to the evaluation of safety. The OECD Group produced after three years a report entitled "Recombinant DNA Safety Considerations", now known as the "Blue Book". This report set out the first international scientific principles and criteria for the safe handling of genetically modified organisms outside of contained laboratory conditions. This pioneering work was considered a milestone in the history of biotechnology after Asilomar where safety guidelines were set up for laboratory work in 1975. The "Blue Book" has had great influence on safety policy worldwide. Its criteria and principles have been adopted by OECD, as well as by non-OECD, countries and appear in most guidelines or legislations for biotechnology safety. Considering that in 1986 the safety assessment of modified organisms to be introduced into the environment was less developed than that of industrial applications, the OECD experts provisionally adopted a case-by-case approach to evaluation of organisms to be used for agricultural or environmental purposes. Such an approach did not assume, however, that some classes of proposals for release could not be excluded or exempted from review by national or other authorities. Furthermore, it was recognised that considerable data that could aid safety assessment were available on the environmental and human health effects of living organisms."
In several regulatory systems, GMO risk assessment has been separated from GMO risk management. As a consequence, risk assessment can be performed on a purely scientific basis, whereas risk management can take additional aspects into consideration, like socioeconomic or ethical questions. The EFSA, the keystone of European Union risk assessment regarding food and feed safety, was established by the European Parliament in 2002, following a series of food scares which undermined consumer confidence in the safety of the food chain. In close collaboration with national authorities and in open consultation with its stakeholders, EFSA provides independent scientific advice and clear communication on existing and emerging risks. In 2004, following several stakeholder consultations and a broad public debate, EFSA published a guidance document for the risk assessment of genetically modified plants and derived food and feed (EFSA, 2004).

After clarifying the scope and legal background in the first chapter, the guidance document describes the overall risk assessment strategy in Chapter 2, including (i) risk assessment, (ii) comparative approach, and (iii) environmental risk assessment and monitoring. Chapter 3 describes the issues to be considered for a comprehensive risk characterization including (i) molecular characterization of the inserts, (ii) assessment of modifications of the agronomic characteristics of the GM plant, and (iii) evaluation of food/feed safety aspects of the GM plant and/or derived food and feed. The risk assessment process is based, case-by-case, on data on composition, toxicity, allergenicity, nutritional value and environmental impact. Chapter 4 summarizes the overall risk characterization process, which may give rise to the need for further activities including post-market monitoring of the GM food/feed and environmental monitoring of the GM plants.

Two principles which have been laid down in the OECD Blue Book are basic for the EFSA guidance document: the case-by-case and the step-by-step approach. In the Blue Book's recommendations specific for environmental and agricultural applications, the two principles have been described as follows: "It is important to evaluate rDNA organisms for potential risk, prior to applications in agriculture and the environment. An independent review of potential risks should be conducted on a caseby-case (case-by-case means an individual review of a proposal against assessment criteria which are relevant to the particular proposal) basis, prior to the application. Development of organisms for agricultural or environmental applications should be conducted in a stepwise fashion, moving, where appropriate, from the laboratory to the growth chamber and greenhouse, to limited field testing and finally, to large-scale field testing." 


\section{REFERENCES}

Casper R., Landsmann J., eds. (1992) Proceedings of the 2nd International Symposium on The Biosafety Results of Field Tests of Genetically Modified Plants and Microorganisms, Biologische Bundesanstalt für Land- und Forstwirtschaft, Braunschweig.
http://www.bba.bund.de/cln_044/nn_913132/DE/Home/ biolsich/gentechnik/taggoslar/Goslar.html

EFSA (2004) Guidance document of the Scientific Panel on Genetically Modified Organisms for the Risk Assessment of Genetically Modified Plants and Derived Food and Feed. The EFSA Journal 99: 1-94.

http://www.efsa.europa.eu/en/science/gmo/gmo_guidance/ 660.html 